\title{
Measuring the Polarity of Conversations between Chatbots and Humans: A Use Case in the Banking Sector
}

\author{
Guillaume Le Noé-Bienvenu \\ OrangeBank \\ 67 Rue Robespierre \\ 93100 Montreuil, France
}

\author{
Damien Nouvel \\ Inalco ERTIM \\ 2 Rue de Lille \\ 75007 Paris, France
}

\author{
Djamel Mostefa \\ OrangeBank \\ 67 Rue Robespierre \\ 93100 Montreuil, France
}

Email: guillaume.lenoe.bienvenu@gmail.com Email: damien.nouvel@inalco.fr Email: djamel.mostefa@orangebank.com

\begin{abstract}
This paper describes a study on opinion analysis applied to both human to chatbot conversations, but also to human to human conversations using data coming from the banking sector. A polarity classifier SVM model applied to conversations provides insights and visualisations of the satisfaction of users at a given time and its evolution. We conducted a study on the evolution of the opinion on the conversations started with the chatbot and then transferred to a human agent. This work illustrates how opinion analysis techniques can be applied to improve the user experience of the customers but also detect topics that generate frustrations with a chatbot or with human experts.
\end{abstract}

\section{INTRODUCTION}

\section{A. Scope and Aim}

O RANGE Bank is a mobile bank launched in late 2017 and for which the main channel of communication with its customers is Djingo, a text chatbot. Available $24 / 7$ by chat, Djingo, is the customers first point of contact. Since the launch of Orange Bank in November 2017, more than 2,5 million conversations have been initiated by our clients with Djingo (an average of 100,000 conversations per month), 50\% of which are handled entirely by the virtual advisor (without any redirection to the Customer Relationship Centre). Since the chatbot is the first point of contact of Orange Bank clients, all chat conversations with a human agent started with Djingo. We are hence able to measure the evolution of the polarity within the same conversation between a customer and Djingo and then between the customer and the human operator.

In this context, opinion mining may be used to deliver in real time an understanding of the customer relationship for a given service. It could also be used to detect annoyance, irritation or angriness at an early stage of the conversation with Djingo in order to quickly redirect the user to a human expert. In this situation, opinion mining is also useful to detect topics and to provide insights about customer's satisfaction.

Our work focuses on the evolution of customer's opinion, both on conversations or messages within conversation. We implemented an opinion detector that has been evaluated, and plugged into the history of online conversations between customers and chatbot or human support desk. This work provides the customer support service visualisations of the evolution of customer's satisfaction depending on themes. The novelty of this paper relies on a comparison of how much the bot vs humans give satisfaction to the customers.

\section{B. State of the Art}

1) Opinion Analysis: Whereas a lot of work has been done in the opinion analysis field, most of it was directed towards product reviews, e.g. identifying the sentiment linked to the aspects of an object or its entities [1], but a few work was done towards written conversations, especially with a chatbot. Reference [2] used the estimation of user satisfaction to improve the learning process of the chatbot. Tools to work on polarity and emotions based on rules such as VADER [3] or SentiWordNet [4] are freely usable, but remain only for the English language. For French, resources are also available, such as the CANÉPHORE Corpus [5], but remain mostly specific to tweets. In this paper, we present a few cases (mostly graphs) in which opinion analysis could help giving valuable information with written talks. We focus on the polarity, defined by [6] as the property of a text being positive, negative or neutral.

2) Text Classification: Text classification is a well known task in NLP, and a reasonably efficient technique to perform it consists of using a TF-IDF [7] representation of the data combined with a support vector machine classifier (SVM) on it. This approach has since be giving satisfactory results. [8], [9], [10]. Deep learning methods can also be used for text classification. In particular, convolutional neural networks obtain very high scores for this task [11], but require more time and examples for training. Also, the winners of many challenges in NLP for the French language used TF-IDF+SVM models as the one used for DEFT 2015 [12] or during the Hackatal 2018 $)$.

${ }^{1}$ https://hackatal.github.io/2018/ 
TABLE I

MOST COMMON ERROR TYPES

\begin{tabular}{|l|l|}
\hline Error type & Example (errors in bold) \\
\hline Diacritics & Je viens deja de vous expliquer mon probleme \\
\hline Case & Comment Recharger son compte ? \\
\hline Punctuation & Ma demande de résiliation $\mathbf{n}$ est toujours pas faite \\
\hline Contraction & Bjr ou envoyer mon RIB ? \\
\hline Typo & Ok je vaiq essayer. Merci \\
\hline Spelling & je n'arive pas a faire foncioné ma carte bancaire \\
\hline
\end{tabular}

\section{The Djingo Chatbot}

Djingo is Orange Bank's conversational agent, available $24 / 7$ for its 3,000 daily users. It is able to understand 390 intentions and has more than 1,000 answers adapted to the user's needs. Djingo is used both as a Frequently Asked Questions (FAQs) system (products marketed e.g. withdrawal fees, time to deliver a cheque book, etc.) and as an assistant to perform actions related to the customer account (ordering a cheque book, blocking the card, etc.). FAQ-oriented answers are usually the same for all customers, whereas requests performing an action trigger an operation that depends on the account.

For example, if a user wishes to order a checkbook, Djingo will check if the user is identified, if there is currently no checkbook order, if the user can order it, and so on. At each step, depending on the elements received through a programmatic interface (APIs), Djingo provides the user with an appropriate answer. During the conversation, themes and intentions are detected by the IBM Watson module. To date, there are about 60 themes: Orange-Bank, app-site-info, app-site-problem, insurance-info, termination insurance, etc. Conversations can include several themes. If the user asks a question that Djingo does not have the answer to, or detects that the user is unable to make himself understood, he suggests that the user should be redirected to an advisor.

\section{OPINIONS FOR MESSAGES AND CONVERSATIONS}

\section{A. Chatbot Corpus}

The corpus used in this article consists of 1,566,060 unique conversations from November 2017 to March 2019, containing $5,775,227$ messages. Most of the messages sent by the users contain a small number of words (around 4.6 words per message) and are often describing the question using simple words. The size of the lexicon is quite important with around $144 \mathrm{k}$ entries due to important number of misspellings and typos.

Table I gives some examples of misspellings errors.

\section{B. Annotation}

As we focus on the polarity of messages, we built a gold-standard, by manually annotating 3,053 randomly picked user messages from the corpus. Each message is considered as positive, negative or neutral, following the 2015 DEFT annotation guide ${ }^{2}$.

The annotation was made by two different annotators, giving a Cohen's kappa coefficient of 0.72. One particular issue during the annotation process was the case of greeting messages. We notice that in our data set, the user uses greetings for $83.96 \%$ of the conversations with a human agent, and only $18.99 \%$ of those with the chatbot. This gives us a clear indication of the behaviour of the user depending on the interlocutor. From an opinion perspective, we then assumed those greetings were positive and annotated them accordingly.

Table II gives examples of annotated data.

TABLE II

EXAMPLE OF ANNOTATED MESSAGES

\begin{tabular}{|l|l|}
\hline Message (translated) & Annotation \\
\hline $\begin{array}{l}\text { Merci orange pour les } 80 \text { euros } \\
\text { Thank you orange for the } 80 \text { euros }\end{array}$ & positive \\
\hline $\begin{array}{l}\text { Merci, bonne soirée } \\
\text { Thank you, have a nice evening }\end{array}$ & positive \\
\hline $\begin{array}{l}\text { OK, super! } \\
\text { Okay, great! }\end{array}$ & positive \\
\hline $\begin{array}{l}\text { Je souhaiterai ouvrir un compte } \\
\text { I'd like you register an account }\end{array}$ & neutral \\
\hline $\begin{array}{l}\text { Savoir si ma demande a été traitée } \\
\text { Find out if my request has been } \\
\text { processed }\end{array}$ & neutral \\
\hline $\begin{array}{l}\text { Quelles sont vos offres pour les } \\
\text { étudiants ? } \\
\text { What are your offers for students? }\end{array}$ & neutral \\
\hline $\begin{array}{l}\text { Cela ne repond pas a la question } \\
\text { This doesn't anwser the question }\end{array}$ & negative \\
\hline $\begin{array}{l}\text { Non merci je suis très contrariée } \\
\text { No, thanks, I'm very upset. }\end{array}$ & negative \\
\hline $\begin{array}{l}\text { Vous servez à rien } \\
\text { You're useless. }\end{array}$ & negative \\
\hline
\end{tabular}

Unsurprisingly, our manual annotations dataset is not balanced: $5.01 \%$ of the messages are positive, $73.96 \%$ of them neutral and $21.03 \%$ negative. This was expected as users usually come with problems and questions regarding bank services and operations. Indeed, the company wants to maximise the satisfaction of users at the end of the interaction, while limiting the number of agents hired for this task.

\section{Classification}

This annotated data set was then divided over a train (4/5) and test parts $(1 / 5)$. The train data was then pre-processed by computing a TF-IDF transformation. We tested several classical machine learning models using the sklearn API [13]. Results are reported in Table III.

TABLE III

PERFORMANCE OF OPINION ClASSIFIER (MACRO)

\begin{tabular}{|l|r|r|r|}
\hline ML classifier & Precision & Recall & F1 \\
\hline SVM & 0.90 & $\mathbf{0 . 8 1}$ & $\mathbf{0 . 8 5}$ \\
\hline MaxEnt & $\mathbf{0 . 9 2}$ & 0.75 & 0.82 \\
\hline MNB & $\mathbf{0 . 9 2}$ & 0.63 & 0.70 \\
\hline SGDClassifier & 0.91 & 0.79 & 0.84 \\
\hline
\end{tabular}

${ }^{2}$ https://deft.limsi.fr/2015/guideAnnotation.fr.php 
TABLE IV

PROPORTION OF MESSAGES AND CONVERSATIONS IN THE CORPUS

\begin{tabular}{|l|r|r|r|r|}
\hline & $\begin{array}{c}\text { Number of } \\
\text { messages }\end{array}$ & $\%$ & $\begin{array}{c}\text { Number of } \\
\text { conversations }\end{array}$ & $\%$ \\
\hline Positive & 460,744 & 3.98 & 190,057 & 7.30 \\
\hline Neutral & $9,903,323$ & 85.50 & $1,746,296$ & 67.07 \\
\hline Negative & $1,218,890$ & 10.52 & 541,549 & 20.80 \\
\hline Mixed & - & - & 125,641 & 4.83 \\
\hline Total & $1,1582,957$ & 100 & $2,603,543$ & 100 \\
\hline
\end{tabular}

As the SVM classifier provides the best F1 score, we ran a grid search on several parameters to optimize this model configuration. We obtained an average $0.85 \mathrm{~F} 1$ macro score (0.91 F1 micro). The neutral class obtains the best score $(0.95$ F1), while positive and negative classes have much lower F1 scores (0.82 and 0.76 , respectively). Those results were obtained using the NLTK TweetTokenizer [14], without any other preprocessing (no lemmatization, case is kept as it is) and linear kernel for the SVM. Finally, the model was used to classify all messages of the corpus.

\section{Conversation Polarity B Y Themes}

\section{A. Rules to Predict Conversations Polarity}

To have a global view of user experience, one needs to compute an opinion score for each conversation. As the data was annotated by messages, simple rules were implemented to predict the polarity of an entire conversation based on the opinion of its messages. A conversation is then:

- neutral when all messages are such,

- positive when at least one of its messages is such and the remaining is neutral or positive,

- negative when at least one of its messages is such and the remaining is neutral or negative,

- mixed otherwise.

Using these simple rules, table IV shows the proportion of messages and conversations by polarity, automatically tagged without manual revision. The rules also allowed us incidentally to get strongly oriented conversations (e.g. a conversation where nearly all of its messages are negative would be very negative).

\section{B. Histogram}

The first representation we get from this labelling is the proportions of the conversation classes (positive, negative, neutral and mixed) depending of the detected themes. Figure 1 shows those proportions for December 2018. For instance, the app_site theme (related to the behaviour of the Bank's application) has more than $50 \%$ of its conversations being negative where the cheque theme remains globally neutral, this can be explained by the fact that this operation is rarely problematic. The representation of polarity gives us a rough idea of where to improve the user's experience. This type of plot can also be drawn for a different time scale (year, day, etc.).

\section{Heatmap}

In the previous section, we presented a way of drawing the proportions of the conversation classes for a particular timelapse. However, this type of plot does not give us information about the evolution of this proportions across a time scale. E.g. on Figure 1, the app_site theme has a strong part of negative conversations but one can wonder if those proportions were similar through the year, whether it was due to a temporary failure, or if it was a general trend.

In order to represent a potential evolution of those proportions, we proposed a heatmap showing this evolution of the opinion by theme. To get a polarity score as a single numerical value for each case, a rule was implemented, consisting of adding the neutral and positive proportions of conversation and subtracting the negative. This was given by the following formula:

$P S(t h, t)=\frac{N(\text { neu }, t h, t)+N(\text { pos }, \text { th }, t)-N(\text { neg }, t h, t)}{N \text { TotalConversations }(\text { th }, t)}$

Where

- th: the theme of the conversation

- $t$ : a date

- $N(p o l, t h, t)$ : the number of conversations of the theme th at time $t$ having the polarity pol (negative, positive or neutral)

- NTotalConversations $(t h, t)$ : the total number of conversations of the theme th at time $t$

Figure 2 reports the heat map from November 2017 to March 2019. The bluer the case is the higher proportion of positive conversations the corresponding theme has. Conversely the red cases indicate negative conversations. One can then watch the changes in the proportions of cases throughout the months. For instance, we clearly see that the Bonus theme in March 2018 had its lowest polarity score, but its polarity score increased in the next few months. As in the previous section, this plot can also be drawn for a different time scale.

\section{Graph of Polarity}

We have then studied the way polarity of messages changes for a single conversation, especially when the user switches from a chatbot to an agent. In order to have a visual output, we converted the polarity (negative, neutral, positive) of each message of the conversation to an integer ( 0 for negative, 1 for neutral, 2 for positive). Table $\mathrm{V}$ shows an example of this conversion. This rule provides us with a list of integers that we can plot on a basic polarity graph, as reported in Figure 3 for a single conversation where each message has its detected polarity mapped on a graph. 


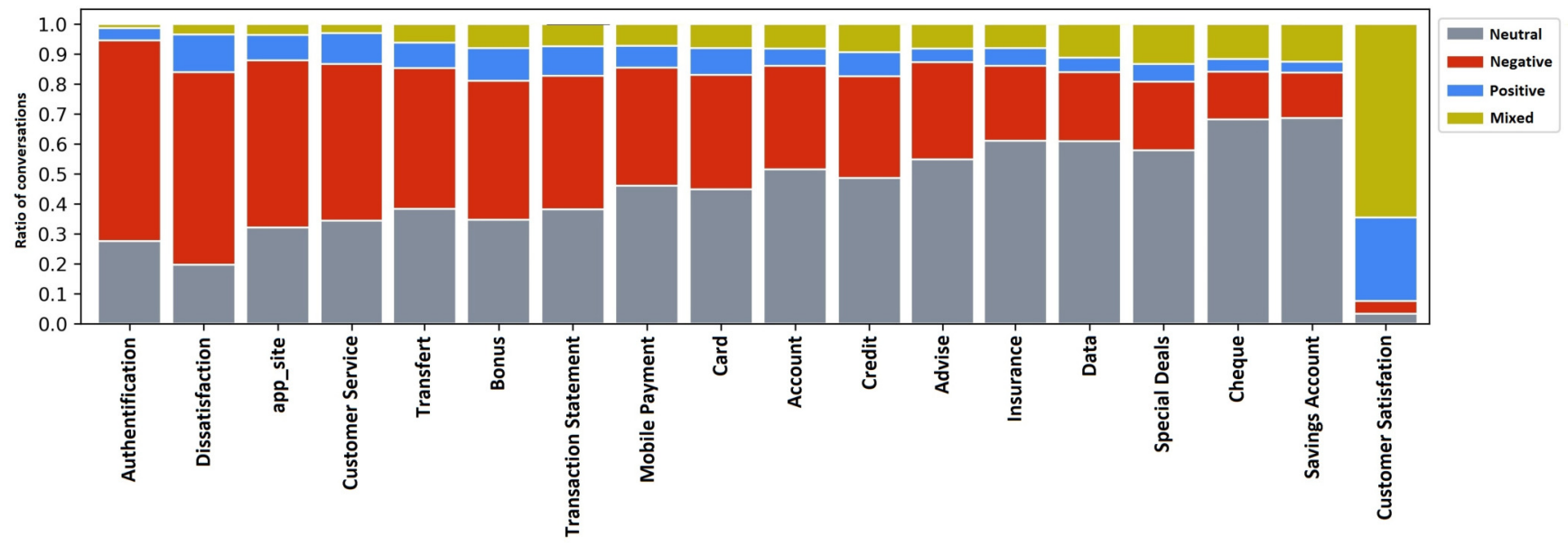

Themes

Fig. 1. Basic polarity histogram

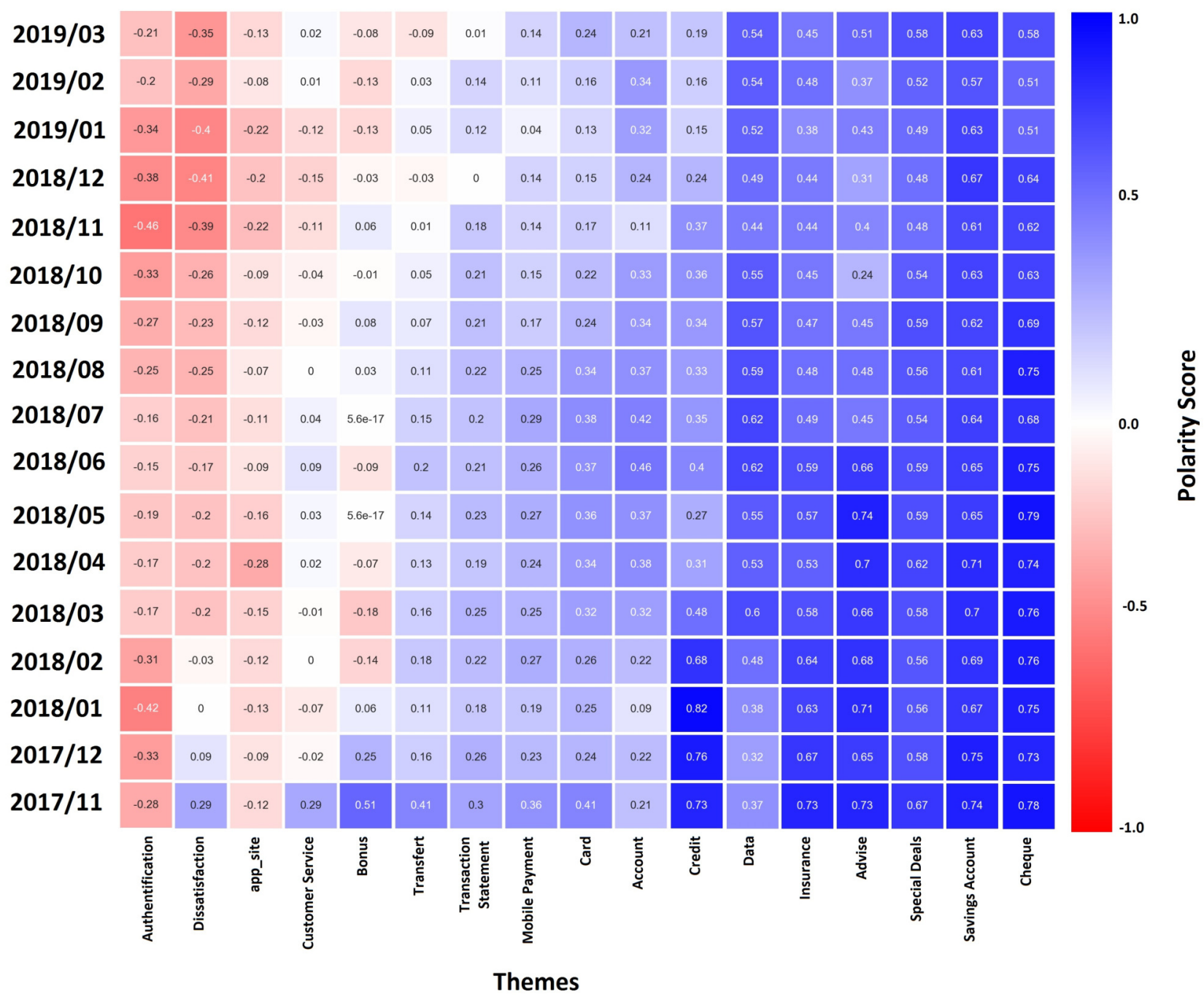

Fig. 2. Heatmap of polarity 


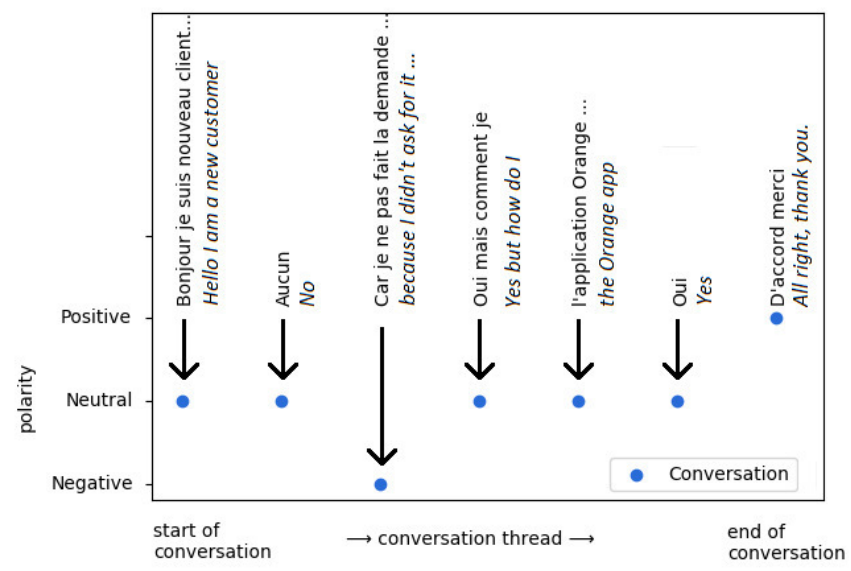

Fig. 3. Single Conversation Polarity Graph

TABLE V

EXAMPLE OF A CONVERSATION CONVERTED TO A GRAPH

\begin{tabular}{|l|l|l|}
\hline Message (translated) & $\begin{array}{c}\text { Predicted } \\
\text { Polarity }\end{array}$ & $\begin{array}{l}\text { Converted } \\
\text { score }\end{array}$ \\
\hline $\begin{array}{l}\text { Bonjour je suis nouveau client mais } \\
\text { je n'ai pas fait la premier conexion } \\
\begin{array}{l}\text { Hello I'm a new customer but } \\
\text { I haven't made the first connection }\end{array}\end{array}$ & negative & 0 \\
\hline $\begin{array}{l}\text { Aucun } \\
\text { None }\end{array}$ & neutral & 1 \\
\hline $\begin{array}{l}\text { Car je ne pas fait la demande de } \\
\text { carte bancaire car je ne pas fait } \\
\text { la demande de carte bancaire } \\
\text { Because I don't apply for a bank } \\
\text { card because I don't apply for } \\
\text { a bank card }\end{array}$ & negative & 0 \\
\hline $\begin{array}{l}\text { Oui mais comment je fait pour me } \\
\text { connecter } \\
\text { Yes, but how do I connect }\end{array}$ & neutral & 1 \\
\hline $\begin{array}{l}\text { 'application Orange Bank } \\
\text { the Orange Bank App }\end{array}$ & neutral & 1 \\
\hline $\begin{array}{l}\text { Oui } \\
\text { Yes }\end{array}$ & neutral & 1 \\
\hline $\begin{array}{l}\text { D'accord merci } \\
\text { All right, thanks. }\end{array}$ & positive & 2 \\
\hline
\end{tabular}

Since the conversations do not have the same length (different number of user messages), we converted the lists of integers representing the polarity of the user messages into lists of floats of fixed size. The size of the output lists can be modified as an optional parameter ${ }^{3}$. We then compute the average of each point of the list. Figure 4 show the result of the output with a padding of dimension 20 .

On Figure 4, we first notice that for both types of users (redirected and non-redirected or full IA), the conversation starts with the same polarity (neutral) on average. After the first third of the conversation, people who are not redirected see the polarity of their conversation stagnate around a value slightly below neutral, while people who will be redirected see the polarity of their conversation decrease until an agent

${ }^{3}$ Code available at https://github.com/GuillaumeLNB/perso/blob/master/ rounding.py

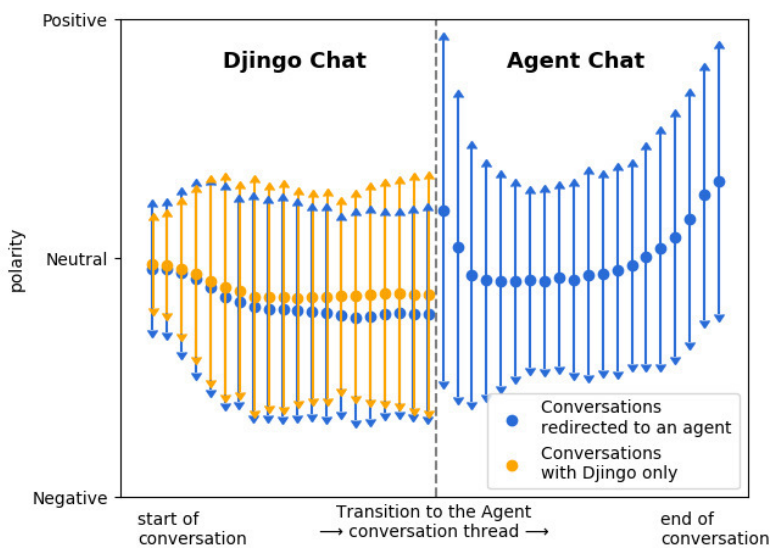

Fig. 4. Polarity graph

takes over. As soon as people are cared for by a counsellor, the polarity of the conversation takes a more positive trend (signs of politeness such as "hello" are labelled as positive and are more present in conversations with a human being). This is followed by a more neutral phase, which generally corresponds to the advisor's information gathering. At the end of the conversation, the trend is clearly becoming positive, we hypothetize that satisfying solutions are being proposed by the human agent.

\section{DISCUSSION}

There are however some limitations to the approaches discussed in this paper. First of all, the classification is based on annotation, and it is quite difficult to annotate into only three polarity classes. In the example: "Mon épouse est décédé et je souhaite réaliser une demande de succession / My wife has died and I want to make a succession request", the user of the conversational agent reports a past event as well as the willingness to take action. However, the part "Mon épouse est décédé / My wife died" would have been annotated as negative, while the part "je souhaite réaliser une demande de succession / I wish to make an estate application" would have been annotated neutral. A new class "positive-negative mix" could have been used as in DEFT $2018^{4}$, but would have required a much more subtle and fine-grained annotation work.

Secondly, polarity is useful information, but does not indicate the subjectivity of the message. There is a significant difference between a user complaining about a particular Orange Bank service (e.g. Ma carte bancaire ne marche pas / My credit card doesn't work, negative polarity) and a dissatisfied user without a specific reason being stated (e.g. Orange c'est vraiment de plus en plus pourri! / Orange is really getting crap!, negative polarity).

Thirdly, the transition from the polarity of the messages to the polarity of the conversation was carried out with a rule-

${ }^{4}$ https://perso.limsi.fr/pap/DEFT2018/annotation_guidelines/index.html 
based approach, creating a mixed class. This class does not take into account the intensity of certain messages. In the example in Table VI, the conversation has a mixed polarity (presence of positive and negative), but remains very negative by the presence of the last message. An annotation at the level of the conversation would probably have classified this conversation as negative, but would not have made a difference between this very negative and a less negative conversation.

TABLE VI

EXAMPLE OF A CONVERSATION CLASSIFIED AS MIXED WHERE IT SHOULD HAVE BEEN NEGATIVE

\begin{tabular}{|l|l|}
\hline Message (translated) & $\begin{array}{c}\text { Predicted } \\
\text { Polarity }\end{array}$ \\
\hline $\begin{array}{l}\text { bonjour, } \\
\text { hello, }\end{array}$ & positive \\
\hline $\begin{array}{l}\text { association loi 1901 peut elle } \\
\text { ouvrir un compte chez vous? } \\
\text { Can a nonprofit association } \\
\text { open an account with you? }\end{array}$ & neutral \\
\hline $\begin{array}{l}\text { compte + association oi 1901 } \\
\text { account + aossociation 1901 [l]aw }\end{array}$ & neutral \\
\hline $\begin{array}{l}\text { je ne parle pas aux robots, connards } \\
\text { I don't talk to robots, assholes. }\end{array}$ & negative \\
\hline
\end{tabular}

Finally, the heatmap display gives us an overview of the evolution of the polarity, but does not detail the reasons of this variation. In addition, we did not find a correlation for all themes between their monthly polarity scores and their redirection rates. We are wondering if this metric is suitable for comparing these data.

\section{CONCLUSION}

In this paper, we have presented several applications of opinion analysis on chatbot conversations. By developing a model for polarity analysis (positive, negative, neutral) using standard machine learning algorithms, we were able to use the data to highlight trends. A real corpus of more than 1.5 million of conversations between Orange bank customers and Djingo was used for this study.

For privacy and confidential reasons, this corpus can not be shared at that time but it may be released in the future after anonymization of all personal data.

This analysis allowed to have a deeper insight of the evolution of the customer satisfaction or dissatisfaction, by topics on a time scale. Polarity mean show the sentiment are generaly more negative for conversation which will be handled by a human agent, what is nice since the human agent raises this polarity to positive values.
This tool makes it possible to obtain a quantification of the customers' opinions on the spot. We foresee that this kind of analysis, merging human and bot answers to a client, will be useful to improve customer relationship management. The key point is to detect when the bot has unsificient capacity to deliver an adequate answer and should pass the dialog to a human agent. It also provides our bank the opportunity to bring out very focused conversations (very positive or negative) from the corpus, to train customer relationship human agents for a better service, therefore this work raises opportunities to improve both the bot and the human agent.

\section{REFERENCES}

[1] B. Liu, "Sentiment Analysis and Opinion Mining", 2012, pp. 11-19.

2] B. Hancock, A. Bordes, P.-E. Mazaré and J. Weston , "Learning from Dialogue after Deployment: Feed Yourself, Chatbot!," CoRR abs/1901.05415, Madison, WI, 2019,

[3] C. J. Hutto and E. Gilbert, "VADER: A Parsimonious Rule-Based Model for Sentiment Analysis of Social Media Text.," 2014

[4] E. Andrea and S. Fabrizio, "SENTIWORDNET: A publicly available lexical resource for opinion mining," in Proceedings of the 5th Conference on Language Resources and Evaluation (LREC), 2006

[5] L. Joseph, E. Morin and S. Peña Saldarriaga, "CANÉPHORE : un corpus français pour la fouille d'opinion ciblée," in Actes de la 22e conférence sur le Traitement Automatique des Langues Naturelles, Caen, France, 2015, pp. 418-424.

[6] L. Zhang and S. Ferrari, "Intensité et polarité : un modèle opératoire articulant plusieurs travaux linguistiques," in Langue française, (num 184), 2014, pp. $35-54$

[7] G. Salton and C. Buckley, "Term-weighting Approaches in Automatic Text Retrieval," in Inf. Process. Manage. vol. 24 num. 5 , Tarrytown, NY, 1988, pp. 513-523.

[8] J. Thorsten, "Text Categorization with Support Vector Machines: Learning with Many Relevant Features," 1998

[9] B. Pang, L. Lee, and S. Vaithyanathan, "Thumbs Up: Sentiment Classification Using Machine Learning Techniques," in Proceedings of the ACL 02 Conference on Empirical Methods in Natural Language Processing vol. 10, Stroudsburg, PA, 2002, pp. 79-86

[10] J. Lilleberg, Y. Zhu, and Y. Zhang, "Support vector machines and Word2vec for text classification with semantic features," in IEEE, 2015/07, pp. 136-140

[11] Y. Kim, "Convolutional Neural Networks for Sentence Classification," in Proceedings of the 2014 Conference on Empirical Methods in Natural Language Processing, EMNLP, Doha, Qatar, 2014, pp. 1746-1751

[12] T. Hamon, A. Fraisse, P. Paroubek, P. Zweigenbaum and C. Grouin, "Analyse des émotions, sentiments et opinions exprimés dans les tweets: présentation et résultats de l'édition 2015 du défi fouille de texte (DEFT)," in Actes de la 22e conférence sur le Traitement Automatique des Langues Naturelles (TALN 2015), 2015, pp. A20.

[13] L. Buitinck, et al., "API design for machine learning software: experiences from the scikit-learn project," in ECML PKDD Workshop: Languages for Data Mining and Machine Learning, Madison, WI, 2013, pp. 108-122.

[14] S. Bird, E. Klein and E. Loper, "Natural Language Processing with Python: Analyzing Text with the Natural Language Toolkit," 2009 